\title{
Adipocyte Cell Death, Fatty Liver Disease and Associated Metabolic Disorders
}

\author{
Akiko Eguchi Ariel E. Feldstein \\ Department of Pediatrics, University of California San Diego, San Diego, Calif., USA
}

\section{Key Words}

Adipocyte cell death · Fatty liver disease $\cdot$ Metabolic disorders

\begin{abstract}
Obesity has reached epidemic proportions in the USA and many other parts of the world. Obesity increases the risk of a number of adverse health conditions including type 2 diabetes, insulin resistance, dyslipidemia, hypertension, and hepatic steatosis. Adipocyte hypertrophy occurs during weight gain and is associated with recruitment of immune cells, mainly macrophages, into the adipose tissue (AT). These cells typically surround a dying or dead adipocyte with the formation of crown-like structures that are present in experimental models of obesity as well as obese humans. The immune infiltration of AT results in increased production of various adipokines, cytokines, and chemokines that play a crucial role in the development of insulin resistance and hepatic steatosis. The pathogenic mechanisms resulting in AT macrophage recruitment are under intense investigation and remain incompletely understood. Recent evidence suggests that various programmed cell death pathways are activated in stressed hypertrophied adipocytes and may result in cell death. These events appear to occur at early stages and be important in triggering the metabolic dysregulation associated with obesity.
\end{abstract}

(2) 2014 S. Karger AG, Basel

$0257-2753 / 14 / 0325-0579 \$ 39.50 / 0$

\section{Introduction}

The prevalence of obesity has continued to increase in the world. Obesity increases the risk of a number of adverse health conditions including type 2 diabetes, dyslipidemia, hypertension, and fatty liver which are grouped under the term metabolic syndrome. Altered adipose tissue (AT) biology has been shown to be a key early event in the development of the metabolic complications of obesity. These changes are mainly characterized by proinflammatory macrophage infiltration of AT resulting in an increased production of a variety of bioactive substances such as cytokines, chemokines, and reactive oxygen species (ROS) that induce insulin resistance and fatty liver. Indeed, in both humans and rodents, inflammatory macrophages accumulate in AT with increasing body weight and preventing the accumulation of macrophages protects against the multiple obesity-related metabolic complications.

The pathogenic mechanisms resulting in inflammatory macrophage infiltration into the AT remain incompletely understood. Infiltrated macrophages are typically found around dying or dead adipocytes with formation of 'crown-like structures' in obese mice and humans [13]. Whether adipocyte death is a late consequence of the AT expansion and inflammation or an upstream event that initiates recruitment of immune cells was a crucial question that up until recently remained unanswered. Re-

\section{KARGER}

E-Mail karger@karger.com

www.karger.com/ddi
Dr. Ariel E. Feldstein

Division of Pediatric Gastroenterology, Hepatology and Nutrition University of California San Diego, 3020 Children's Way, MC 5030 San Diego, CA 92103-8450 (USA)

E-Mail afeldstein@ucsd.edu 
Fig. 1. During weight gain and obesity, hypertrophied adipocytes release adipokines and chemokines that result in recruitment of immune cells, mainly macrophages that release further chemokines/cytokines perpetuating a 'pro-inflammatory' state that induces local and systemic insulin resistance. Hypertrophied, insulin-resistant adipocytes release FFAs into the circulation that accumulate in various organs and tissues such as the liver resulting in hepatic steatosis and metabolic deregulation.

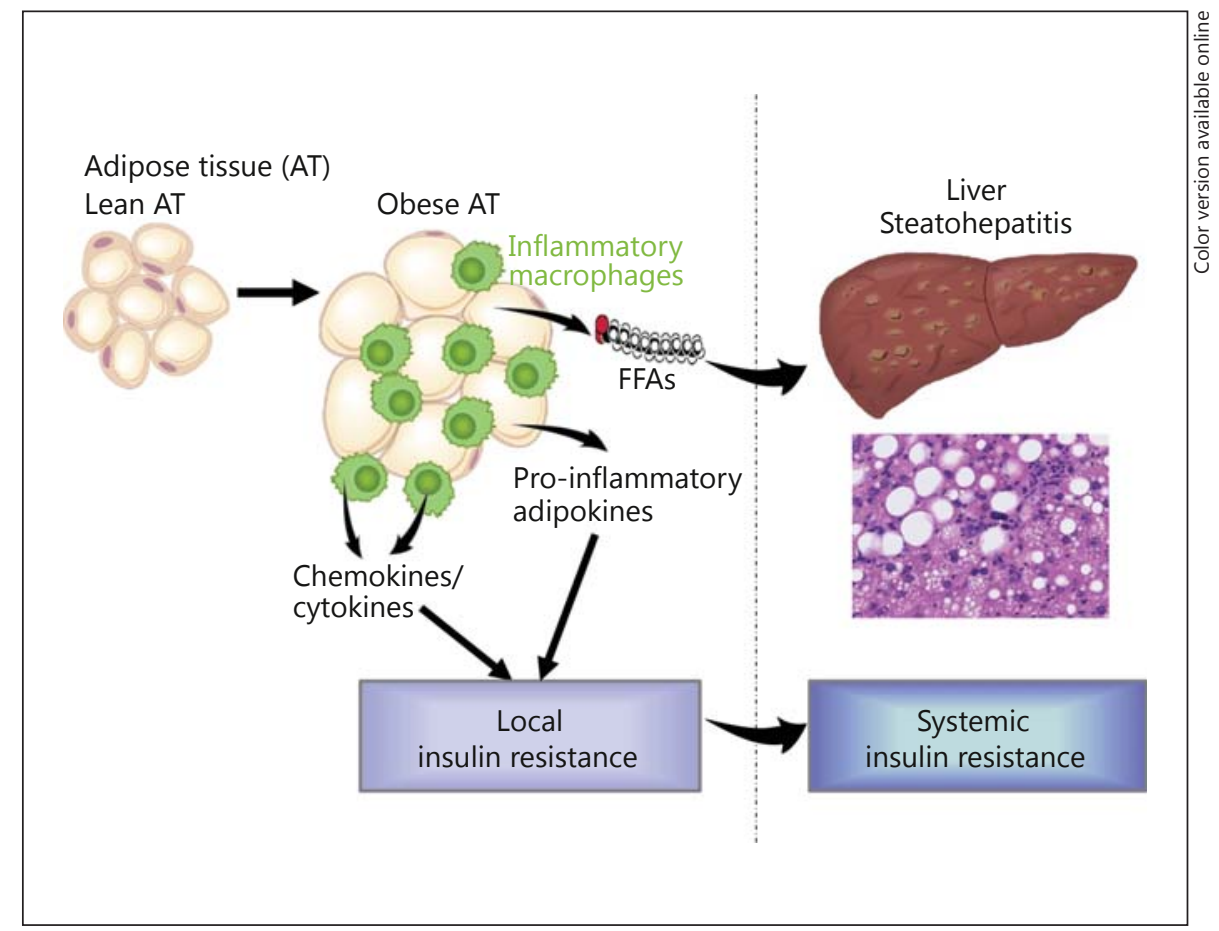

cently, we have demonstrated that programmed cell death initiated by lysosomal permeabilization and mitochondrial dysfunction occurs during the early stages of hypertrophy in adipocytes resulting in the acceleration of infiltrated inflammatory macrophages and the development of metabolic disorders $[4,5]$. In addition, studies have recently shown that endoplasmic reticulum (ER) stress signals are activated in the early stage of hypertrophic adipocytes and these events can result in mitochondrial-dependent cell death [6].

\section{Metabolic Disorders of Obesity: The Role of Adipose Tissue}

The metabolic disorders associated with obesity are clustered under the name metabolic syndrome. Growing evidence supports a key role for altered AT biology as a key denominator for these metabolic complications. During weight gain, adipocytes are gradually hypertrophied, associated with recruitment of bone marrow-derived macrophages into the AT and increased levels of various pro-inflammatory adipokines, chemokines/cytokines resulting in local insulin resistance and development of systemic insulin resistance (fig. 1) [7]. These proinflammatory adipokines and chemokines/cytokines are also released into the bloodstream affecting distant target tissues and organs [8]. Concomitantly, free fatty acids (FFAs) are released into the bloodstream from hypertrophied adipocytes via increased lipolysis as a result of AT insulin resistance, and delivered to other organs such as liver and muscle [9]. In the liver, these FFAs are taken up by hepatocytes and may induce lipotoxicity resulting in activation of distinct biochemical cascades of cell death (fig. 1). Non-alcoholic fatty liver disease (NAFLD) has increased dramatically mainly in the Western world, affecting about $30 \%$ of the American adult population, and is closely associated with obesity. Non-alcoholic steatohepatitis (NASH) is a more serious form of NAFLD which is characterized by hepatic steatosis in association with hepatocellular injury and inflammation with various degrees of liver fibrosis [10]. Furthermore, approximately $10-25 \%$ of NASH patients can progress to cirrhosis and its associated complications including hepatocellular carcinoma or liver failure, requiring a liver transplantation [11, 12].

Thus, changes in adipocyte biology appear as key initiators and early events leading to fat accumulation in the liver, supporting the importance of dissecting the alterations in adipocytes during weight gain to potentially identify early upstream targets for treatment of fatty liver disorders. 


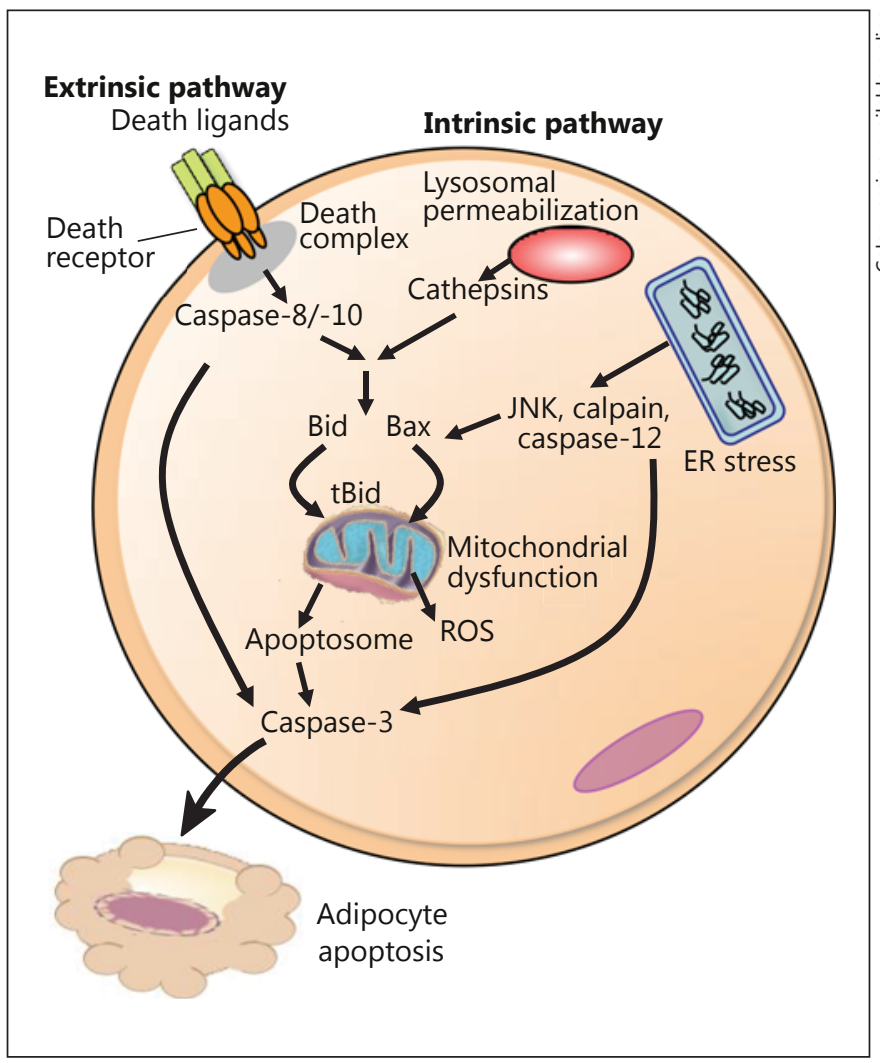

Fig. 2. Extrinsic and intrinsic pathways of cell death may activate during adipocyte hypertrophy and result in adipocyte cell death. In the extrinsic pathway, death receptors including Fas, TNF-R1 and TRAIL-Rs are engaged by their natural ligands. These receptors trigger intracellular cascades that activate death-inducing proteolytic enzymes, especially caspases. In the intrinsic pathway, organelle stress such as lysosomal permeabilization or ER stress activates pro-apoptotic proteins such as Bid or Bax resulting in mitochondrial dysfunction with release of several pro-apoptotic proteins into the cytosol including cytochrome $\mathrm{c}$, which then forms an activation complex with Apaf-1 and caspase-9, the apoptosome.

\section{Adipocyte Cell Death}

It is well characterized that morphologically appearing dying or dead adipocytes surrounded by infiltrated inflammatory macrophages, so-called crown-like structures, are present in the AT during weight gain and the number of crown-like structures increases overtime [3]. However, the molecular and biochemical pathways initiating cell death in adipocytes has until recently remained unstudied. Recent studies reported that both caspase-dependent and -independent cell death occurs in hypertrophied adipocytes in experimental murine models of obesity and further dissected the signaling pathways that initiate this process (fig. 2) $[4,5,13]$.

Adipocyte Cell Death, Fatty Liver Disease and Associated Metabolic Disorders

\section{Lysosomal-Mitochondrial Axis}

Lysosomes play a central role in both necrotic and apoptotic cell death. Lysosomal permeabilization is an early event during apoptosis and it typically precedes other features characteristic of the cell death processes [14]. A total of 11 human cathepsins have been identified and cathepsin B (cysteine protease) as well as cathepsin $\mathrm{D}$ (aspartic protease) are the two most abundant proteases in the lysosome. During lysosomal permeabilization, the active form of cathepsins is released into the cytosol and induces activation of the pro-apoptotic Bcl-2 family protein such as Bax and Bid. Sequentially, these activated pro-apoptotic proteins move to the mitochondria outer membrane and trigger mitochondrial dysfunction with formation of the apoptosome [cytochrome c, apoptotic-protein activation factor-1 (Apaf-1), and caspase-9] resulting in cellular apoptosis through caspase- 3 activation. In addition, the ROS produced by mitochondrial dysfunction can also feedback to the lysosome resulting in further lysosomal breakdown and cell damage [15].

Several studies suggest that cathepsins may play an important role in the altered adipocyte biology in obesity. Masson et al. [16] reported that cathepsin D (CTSD) mRNA levels are upregulated in AT from obese humans, as well as in adipocytes from the obese mouse fed a highfat diet (HFAT) at age 30 weeks. They also proposed a key role of CTSD in the control of adipogenesis in murine pre-adipocytes during differentiation using RNAi technology for CTSD knockdown. Feng et al. [17] reported that CTSD activity is increased in mice fed a HFAT diet for 4 and 8 weeks. Furthermore, we have demonstrated that a lysosomal-mitochondrial axis involving cathepsins and $\mathrm{Bax} / \mathrm{Bid}$ is a potential mechanism for adipocyte apoptosis and macrophage infiltration during obesity $[5,18]$. We first demonstrated that FFAs induced lysosomal permeabilization with cathepsin activation resulting in adipocyte apoptosis through mitochondrial dysfunction in vitro. Notably, adipocyte apoptosis was protected when the cells were incubated with FFAs plus a cathepsin B (CTSB) inhibitor due to inhibition of lysosomal permeabilization. In obese mouse, CTSB and CTSD activation were detected in epididymal AT (eAT) as early as 2 weeks after initiation of a HFAT diet and associated with a slight increase in adipocyte apoptosis. After 6 weeks of HFAT diet, CTSB and CTSD activation were further increased and preceded the activation of pro-apoptotic proteins Bid and Bax that act directly on mitochondria to induce mitochondrial dysfunction, subsequently increase ROS pro- 
duction, and release several mitochondrial intermembrane space proteins including cytochrome $\mathrm{c}$ into the cytosol. As a result of lysosomal permeabilization and mitochondrial dysfunction, a significant increase in adipocyte cell death could be detected and was associated with an increase in macrophage infiltration. Notably, genetic inactivation of CTSB in CTSB knockout mice $\left(\mathrm{CTSB}^{-/-}\right)$resulted in a markedly decreased recruitment of macrophages to eAT and inflammatory cytokines induced by a HFAT diet, and protected against ROS production resulting in reduction of adipocyte cell death. In conclusion, these studies identify lysosomal cathepsins as the potential key mediator of adipocyte cell death during obesity.

\section{Endoplasmic Reticulum}

ER is a central organelle as the location of protein folding, protein maturation, lipid synthesis, and storage of free calcium. ER has a key control system by which only properly folded proteins are delivered to the extracellular environment through the Golgi compartment. Multiple physiological and pathological conditions can interfere with ER quality control and lead to an accumulation of unfolded proteins in the ER. Such an increase of unfolded proteins is termed ER stress and can have deleterious consequences for the cell. To restore normal cell function from ER stress, the cells activate the unfolded protein response (UPR) that is mediated by ER intracellular signal transduction pathways involving induction of IRE1 (ER transmembrane kinase/endoribonuclease), PERK (PKRlike ER kinase), and ATF-6 (activating transcription factor 6) [19]. However, when the ER stress exceeds the processing capacity of the UPR, the UPR may trigger cell death via apoptosis through multiple pathways such as JNK (c-Jun N-terminal kinase), caspase-12 or mitochondrial dysfunction [20,21].

Recent studies have shown that ER stress is increased in adipocytes from obese mice, both in genetically obese (ob/ob) or diet-induced obese mice [22]. The expression of GRP78 (78-kDa glucose-regulated/binding immunoglobulin protein), a central component of the UPR, PERK phosphorylation, and JNK activity were all significantly increased in AT of obese mice compared to lean controls. The Boden and Merali group has reported the upregulation of ER stress in obese humans compared to lean individuals [23]. They showed that the expression of several UPR stress-related proteins including JNK-1 was increased in obese patients. Furthermore, the Lionetti and Barletta [6] group reported that the hypertrophic adipocyte is under ER stress resulting in the activation of meta- bolic pathways that trigger insulin resistance, release macrophage chemoattractant proteins and induce adipocyte death.

\section{Death Receptors}

The extrinsic pathway of cell death is initiated by death receptors including Fas (CD95 or APO-1), TNFR1 and TRAIL-Rs. When engaged by their natural ligands, these receptors trigger intracellular cascades that activate death-inducing proteolytic enzymes, especially caspases. Fas is a glycosylated protein that is widely expressed and is activated by binding of Fas ligand (FasL) resulting in receptor trimerization and the formation of the death-inducing signaling complex (DISC) [24]. The DISC contains a Fas-associated protein with death domain (FADD). FADD activates caspase- 8 following activation of executioner caspases (caspase-3, -6 and -7), either directly (type I pathway) or indirectly through mitochondrial dysfunction (type II pathway) [25]. Mitochondrial dysfunction results in release of several proapoptotic proteins into the cytosol including cytochrome c, which then forms an activation complex with Apaf-1 and caspase-9, the apoptosome. In the death complex of TNF-R1 with TNF- $\alpha$, TNF-R1 engages TNF receptorassociated protein with death domain (TRADD), receptor interacting protein 1 (RIP1) and TNF receptor-associated factor 2 (TRAF2) to form the so-called complex I [26]. Complex I binds to FADD, resulting in caspase-8 activation and cell death.

Recent studies showed that serum concentration of both Fas and FasL were associated with metabolic disorders, type 2 diabetes, hypertension, and cardiovascular disease [27]. In adipocytes, the Wabitsch group [28] has reported that human adipocytes express FasL, TNF-R1, TRAIL-R1 (TNF-related apoptosis-inducing ligand receptor 1), and TRAIL-R2. We have also demonstrated that Fas and FasL protein expression are significantly increased in the hypertrophied AT of obese mice fed a HFAT diet compared to control diet [4]. Fas and FasL complex activates Fas signaling pathway resulting in activation of caspase-8, increase of pro-apoptotic proteins (tBid and Bax), decrease of anti-apoptotic protein (Bcl$\mathrm{X}_{\mathrm{L}}$ ), and activation of caspase-3. As a result, adipocyte apoptosis occurs associated with increase of macrophage infiltration and upregulation of pro-inflammatory genes. As mitochondrial dysfunction is a key event to control apoptosis mediated by both extrinsic and intrinsic pathways, we investigated whether inhibition of mitochondrial dysfunction would reduce the adipocyte apoptosis in Bid knockout (Bid KO) mouse fed a HFAT diet. As 
Fig. 3. Adipocyte cell death occurs at early stage during AT expansion and adipocyte hypertrophy associated with obesity and accelerates recruitment of immune cells to AT, predominantly macrophages and the downstream events leading to insulin resistance and hepatic steatosis. Identifying the molecular and biochemical mechanisms initiating adipocyte cell death may result in the discovery of novel therapeutic targets for treatment of the various metabolic complications associated with obesity.

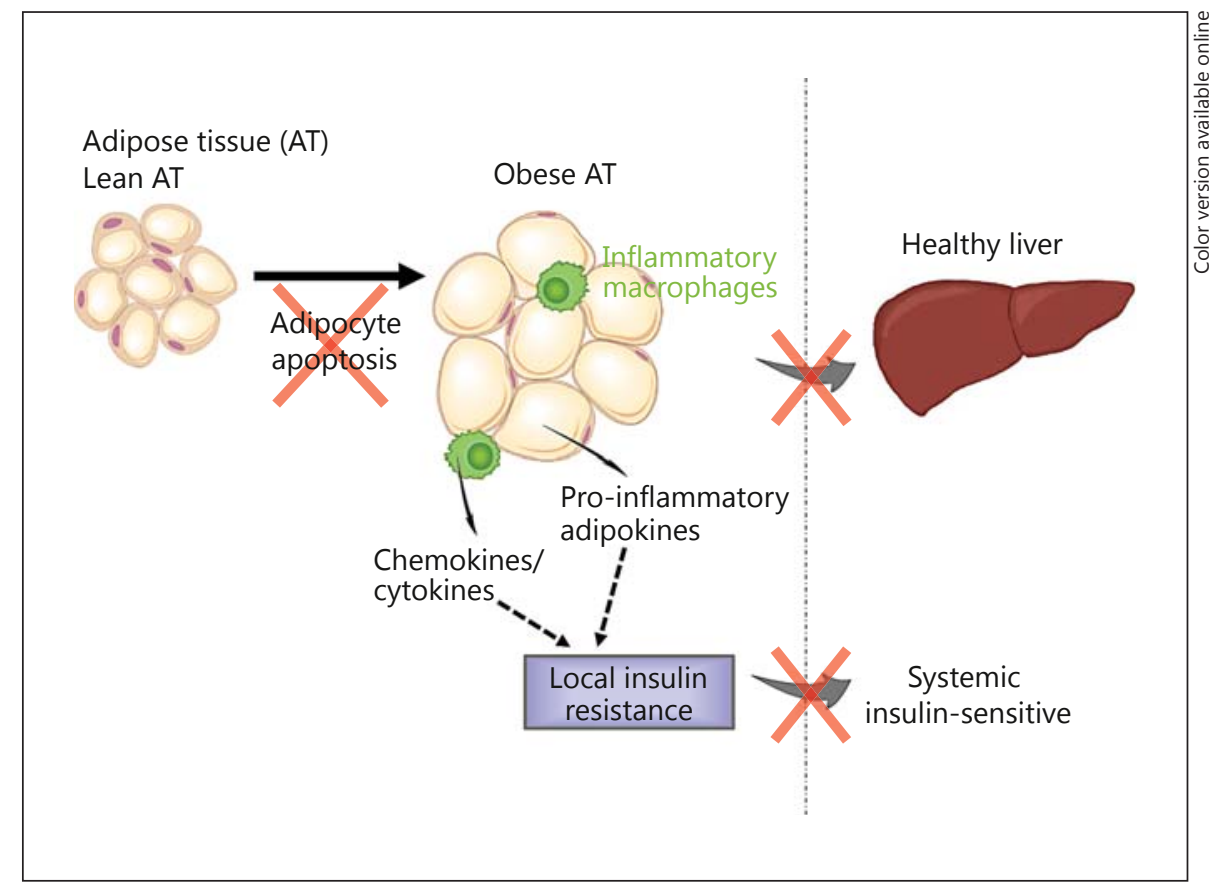

expected, adipocyte apoptosis was significantly reduced in Bid KO mice due to inhibition of mitochondrial dysfunction associating with decrease recruitment of infiltrating macrophages and reduce activation of pro-inflammatory genes compared to wild-type (WT) mice fed a HFAT diet.

\section{NLR Inflammsomes and Caspase-1-Mediated Cell Death}

Inflammasomes are multiprotein cytoplasmic complexes that serve as pattern recognition receptors and have emerged as key mediators of inflammation, immunity, and cell death. They are composed of one of several NLR and PYHIN proteins, including NLRP1, NLRP3, NLRC4 and AIM2.1 Inflammasome activation regulates the cleavage of effector pro-inflammatory cytokines such as pro-IL- $1 \beta$ and pro-IL-18 through the processing and activation of caspase-1. We, and others, have recently shown that hepatic caspase- 1 activation occurs in both bone marrow-derived Kupffer cells (macrophages) and hepatocytes during the development of NASH and alcoholic steatohepatitis (ASH) and this activation appears to be mediated through the NLRP3 inflammasome [29]. We have recently shown that NLRP3 activation is sufficient to induce hepatic inflammation and fibrosis [30]. Furthermore, we demonstrated that hepatocytes may undergo pyroptotic cell death, a novel form of programmed cell death that differs from other types of cell death in that is dependent on caspase-1 activation. More recently, Giordano et al. [31] demonstrated that hypertrophied adipocytes in obese mouse models may also undergo pyroptosis and this could be an important mechanism contributing to macrophage recruitment and perpetuating the inflammatory process in obese AT.

\section{Inhibition of Adipocyte Cell Death as an Approach for Treatment of Metabolic Disorders}

The various studies reviewed in the above sections suggest that targeting adipocyte cell death may be an attracting therapeutic approach for fatty liver disease and other metabolic complications of obesity. As a proof of concept, we tested the hypothesis that Bid suppression may result in improvement of metabolic complications associated with obesity. We initially focused on the effects on insulin sensitivity and hepatic steatosis using WT mice or global Bid KO mice fed a HFAT diet [4]. As expected, WT mice fed a HFAT diet developed significantly increased serum FFA levels, systemic insulin resistance and hepatic steatosis associated with a significant increase of hepatic TG levels. On the other hand, Bid $\mathrm{KO}$ mice fed the HFAT diet were protected from development of systemic insulin resistance and diet-induced hepatic steatosis. These data suggest that adipo- 
cyte Bid activation and cell death is an early event that contributes to the development of fatty liver and insulin resistance associated with obesity and identifies a novel potential target for therapeutic strategies for these conditions.

\section{Conclusion}

Adipocyte cell death initiated by activation of death receptors on the cell surface and/or organelle stress occurs at an early stage during hypertrophy of adipocytes and expansion of AT associated with obesity. Subsequently, hypertrophied adipocytes release a number of adipokines and chemokines that results in immune cell infiltration of AT, composed predominantly of macrophages that induce further release of cytokines/chemokines perpetuating the adipocyte stress and inducing lo$\mathrm{cal}$ and systemic metabolic dysregulation. The inhibition of specific signaling pathways activated during cell stress such as lysosomal permeabilization or mitochondrial dysfunction may protect adipocytes and prevent cell death and subsequent downstream events. These data have uncovered novel aspects of adipocyte biology during obesity. Future studies to further dissect the molecular and biochemical events occurring during adipocyte hypertrophy are warranted and may result in the discovery of novel therapeutic targets for treatment of the various metabolic complications associated with obesity (fig. 3).

\section{Acknowledgement}

This work was supported by NIH grants (RO1DK076852) and (RO1DK082451) to A.E.F.

\section{Disclosure Statement}

The authors have no conflicts of interest to disclose.

\section{References}

1 Strissel KJ, Stancheva Z, Miyoshi H, Perfield JW II, DeFuria J, Jick Z, Greenberg AS, et al: Adipocyte death, adipose tissue remodeling, and obesity complications. Diabetes 2007;56: 2910-2918.

-2 Murano I, Barbatelli G, Parisani V, Latini C, Muzzonigro G, Castellucci M, Cinti S: Dead adipocytes, detected as crown-like structures, are prevalent in visceral fat depots of genetically obese mice. J Lipid Res 2008;49:15621568.

-3 Cinti S, Mitchell G, Barbatelli G, Murano I, Ceresi E, Faloia E, Wang SP, et al: Adipocyte death defines macrophage localization and function in adipose tissue of obese mice and humans. J Lipid Res 2005;46:2347-2355.

4 Alkhouri N, Gornicka A, Berk MP, Thapaliya S, Dixon LJ, Kashyap S, Schauer PR, et al: Adipocyte apoptosis, a link between obesity, insulin resistance, and hepatic steatosis. J Biol Chem 2010;285:3428-3438.

-5 Gornicka A, Fettig J, Eguchi A, Berk MP, Thapaliya S, Dixon LJ, Feldstein AE: Adipocyte hypertrophy is associated with lysosomal permeability both in vivo and in vitro: role in adipose tissue inflammation. Am J Physiol Endocrinol Metab 2012;303:E597-E606.

-6 Lionetti L, Mollica MP, Lombardi A, Cavaliere G, Gifuni G, Barletta A: From chronic overnutrition to insulin resistance: the role of fat-storing capacity and inflammation. Nutr Metab Cardiovasc Dis 2009;19:146-152.
7 Ouchi N, Parker JL, Lugus JJ, Walsh K: Adipokines in inflammation and metabolic disease. Nat Rev Immunol 2011;11:85-97.

-8 Lau DC, Dhillon B, Yan H, Szmitko PE, Verma S: Adipokines: molecular links between obesity and atheroslcerosis. Am J Physiol Heart Circ Physiol 2005;288:H2031-H2041.

9 Frayn KN, Arner P, Yki-Jarvinen H: Fatty acid metabolism in adipose tissue, muscle and liver in health and disease. Essays Biochem 2006;42:89-103.

10 Feldstein AE, Canbay A, Angulo P, Taniai M, Burgart LJ, Lindor KD, Gores GJ: Hepatocyte apoptosis and Fas expression are prominent features of human nonalcoholic steatohepatitis. Gastroenterology 2003;125:437-443.

11 Adams LA, Lymp JF, St Sauver J, Sanderson SO, Lindor KD, Feldstein A, Angulo P: The natural history of nonalcoholic fatty liver disease: a population-based cohort study. Gastroenterology 2005;129:113-121.

12 Ascha MS, Hanouneh IA, Lopez R, Tamimi TAR, Feldstein AF, Zein NN: The incidence and risk factors of hepatocellular carcinoma in patients with nonalcoholic steatohepatitis. Hepatology 2010;51:1972-1978.

13 Feldstein AE, Gores GJ: Apoptosis in alcoholic and nonalcoholic steatohepatitis. Front Biosci 2005;10:3093-3099.

14 Guicciardi ME, Leist M, Gores GJ: Lysosomes in cell death. Oncogene 2004;23:2881-2890.
15 Boya P, Andreau K, Poncet D, Zamzami N, Perfettini JL, Metivier D, Ojcius DM, et al: Lysosomal membrane permeabilization induces cell death in a mitochondrion-dependent fashion. J Exp Med 2003;197:1323-1334.

16 Masson O, Prebois C, Derocq D, Meulle A, Dray C, Daviaud D, Quilliot D, et al: Cathepsin-D, a key protease in breast cancer, is upregulated in obese mouse and human adipose tissue, and controls adipogenesis. Plos One 2011;6:e16452.

17 Feng DR, Tang Y, Kwon H, Zong HH, Hawkins M, Kitsis RN, Pessin JE: High-fat diet induced adipocyte cell death occurs through a cyclophilin D intrinsic signaling pathway independent of adipose tissue inflammation. Diabetes 2011;60:2134-2143.

18 Eguchi A, Feldstein AE: Lysosomal cathepsin D contributes to cell death during adipocyte hypertrophy. Adipocyte 2013;2:170-175.

19 Ron D, Walter P: Signal integration in the endoplasmic reticulum unfolded protein response. Nat Rev Mol Cell Biol 2007;8:519529.

20 Breckenridge DG, Germain M, Mathai JP, Nguyen M, Shore GC: Regulation of apoptosis by endoplasmic reticulum pathways. Oncogene 2003;22:8608-8618.

21 Li JZ, Lee B, Lee AS: Endoplasmic reticulum stress-induced apoptosis - multiple pathways and activation of $\mathrm{p} 53$-upregulated modulator of apoptosis (PUMA) and NOXA by p53. J Biol Chem 2006;281:7260-7270. 
-22 Ozcan U, Cao Q, Yilmaz E, Lee AH, Iwakoshi NN, Ozdelen E, Tuncman G, et al: Endoplasmic reticulum stress links obesity, insulin action, and type 2 diabetes. Science 2004;306: 457-461.

23 Boden G, Duan X, Homko C, Molina EJ, Song $\mathrm{W}$, Perez $\mathrm{O}$, Cheung $\mathrm{P}$, et al: Increase in endoplasmic reticulum stress-related proteins and genes in adipose tissue of obese, insulin-resistant individuals. Diabetes 2008;57:24382444.

24 Peter ME, Krammer PH: The CD95(APO-1/ Fas) DISC and beyond. Cell Death Differ 2003;10:26-35.

25 Scaffidi C, Fulda S, Srinivasan A, Friesen C, Li F, Tomaselli KJ, Debatin KM, et al: Two CD95 (APO-1/Fas) signaling pathways. EMBO J 1998;17:1675-1687.
26 Micheau O, Tschopp J: Induction of TNF receptor I-mediated apoptosis via two sequential signaling complexes. Cell 2003;114:181-190.

27 Cosson E, Bringuier AF, Paries J, Guillot R, Vaysse J, Attali JR, Feldmann G, et al: Fas/FasLigand pathway is impaired in patients with type 2 diabetes. Influence of hypertension and insulin resistance. Diabetes Metab 2005;31: 47-54.

28 Fischer-Posovszky P, Tornqvist H, Debatin KM, Wabitsch M: Inhibition of death receptor-mediated apoptosis in human adipocytes by the insulin-like growth factor I (IGF-I)/ IGF-I receptor autocrine circuit. Endocrinology 2004;145:1849-1859.
29 Dixon LJ, Flask CA, Papouchado BG, Feldstein AE, Nagy LE: Caspase- 1 as a central regulator of high-fat diet-induced non-alcoholic steatohepatitis. PLoS One 2013;8:e56100.

30 Wree A, Eguchi A, McGeough MD, Pena CA, Johnson CD, Canbay A, Hoffman HM, et al: NLRP3 inflammasome activation results in hepatocyte pyroptosis, liver inflammation and fibrosis. Hepatology 2013, Epub ahead of print.

31 Giordano A, Murano I, Mondini E, Perugini J, Smorlesi A, Severi I, Barazzoni R, et al: Obese adipocytes show ultrastructural features of stressed cells and die of pyroptosis. J Lipid Res 2013;54:2423-2436. 